Hans Benker

\title{
Differentialgleichungen mit MATHCAD und MATLAB
}

Mit 33 Abbildungen

暨 Springer 


\section{Inhaltsverzeichnis}

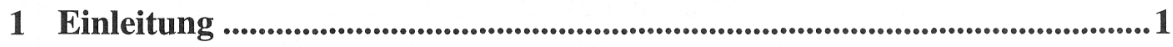

1.1 Differentialgleichungen in Technik, Natur- und

Wirtschaftswissenschaften.................................................... 2

1.2 Lösung von Differentialgleichungen ......................................... 3

1.2.1 Einführung................................................................... 3

1.2.2 Anwendung von MATHCAD und MATLAB .......................... 3

1.2.3 Anwendung weiterer Programmsysteme................................ 5

1.3 Hinweise zur Benutzung des Buches........................................ 5

2 Differenzengleichungen .................................................................................. 7

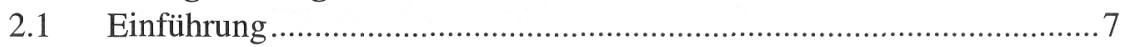

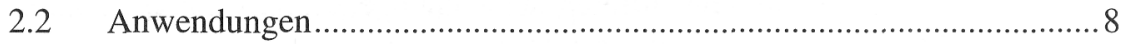

2.3 Lösungsmethoden................................................................ 10

2.3.1 Lineare Differenzengleichungen ...................................... 10

2.3.2 Anwendung der z-Transformation ..................................... 13

2.3.3 Anwendung von MATHCAD und MATLAB ....................... 14

3 Differentialgleichungen ................................................................... 17

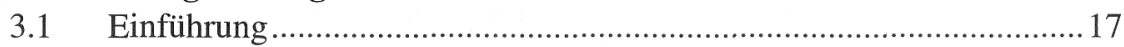

3.2 Gewöhnliche Differentialgleichungen........................................... 18

3.3 Partielle Differentialgleichungen .............................................. 18

3.4 Systeme von Differentialgleichungen......................................... 18

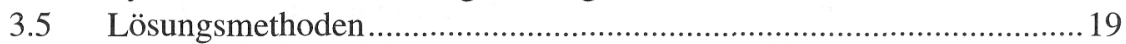

3.5.1 Exakte Lösungsmethoden............................................. 20

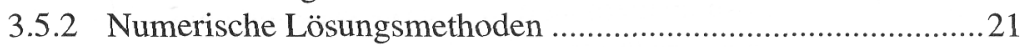

3.5.3 Anwendung von MATHCAD und MATLAB .......................22

4 Gewöhnliche Differentialgleichungen ...................................................... 23

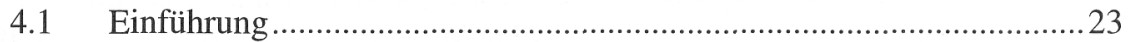

4.2 Anwendungen ......................................................................... 24

4.3 Anfangs-, Rand- und Eigenwertaufgaben......................................25

4.4 Existenz und Eindeutigkeit von Lösungen ....................................2 27

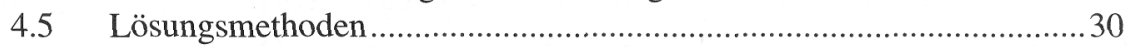

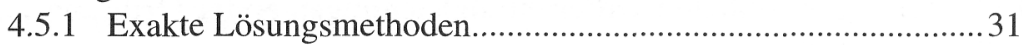

4.5.2 Numerische Lösungsmethoden .......................................... 31

4.5.3 Anwendung von MATHCAD und MATLAB ........................ 31 
5 Gewöhnliche Differentialgleichungen erster Ordnung.....................................33

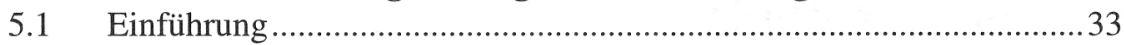

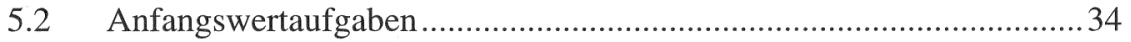

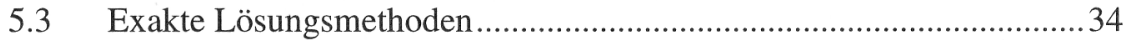

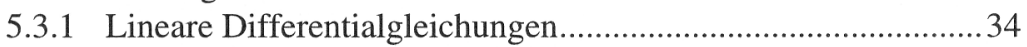

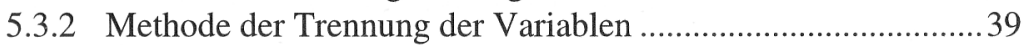

5.3.3 Homogene Differentialgleichungen ...........................................4

5.3.4 Exakte Differentialgleichungen................................................. 41

5.3.5 Bernoullische Differentialgleichungen.......................................4

5.3.6 Riccatische Differentialgleichungen .........................................45

5.3.7 Anwendung von MATHCAD und MATLAB ……................... 45

6 Gewöhnliche Differentialgleichungen zweiter Ordnung ...............................47

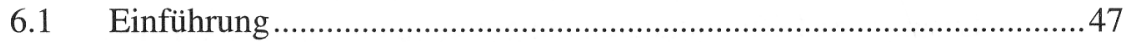

6.2 Anfangs-, Rand- und Eigenwertaufgaben.........................................48

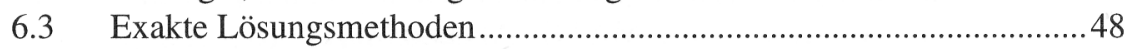

6.3.1 Zurückführung auf Differentialgleichungen erster Ordnung.....48

6.3.2 Lineare Differentialgleichungen.................................................51

6.3.3 Besselsche Differentialgleichungen ..........................................54

6.3.4 Hypergeometrische Differentialgleichungen.............................56

6.3.5 Legendresche Differentialgleichungen.....................................57

6.3.6 Sturmsche Randwertaufgaben ..................................................58

6.3.7 Sturm-Liouvillesche Eigenwertaufgaben ..................................62

6.3.8 Anwendung von MATHCAD und MATLAB ...........................65

7 Gewöhnliche Differentialgleichungen n-ter Ordnung ....................................667

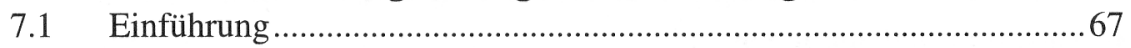

7.2 Anfangs-, Rand- und Eigenwertaufgaben......................................... 70

7.3 Lineare Differentialgleichungen .........................................................72

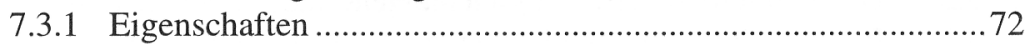

7.3.2 Konstante Koeffizienten...........................................................

7.3.3 Euler-Cauchysche Differentialgleichungen................................77

7.3.4 Spezielle Lösungen inhomogener Differentialgleichungen........81

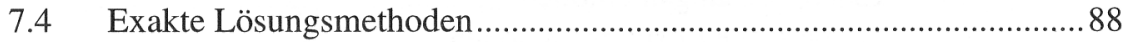

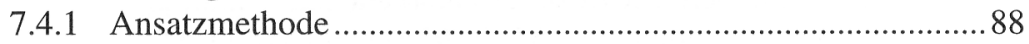

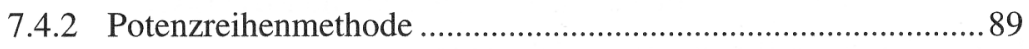

7.4.3 Anwendung der Laplacetransformation ...................................92

7.4.4 Methode der Greenschen Funktionen.....................................100

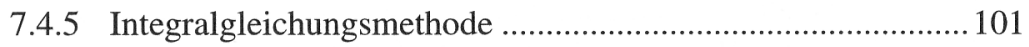

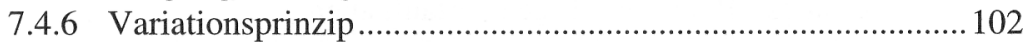

7.4.7 Anwendung von MATHCAD und MATLAB ………….......... 104

8 Systeme gewöhnlicher Differentialgleichungen erster Ordnung ...............107

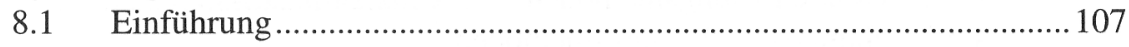

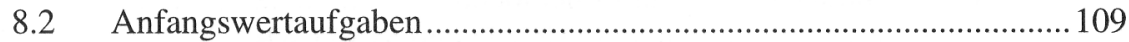

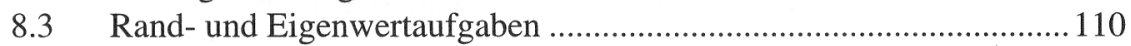

8.4 Lineare Differentialgleichungssysteme ............................................ 111 
8.4.1 Eigenschaften ........................................................... 112

8.4.2 Konstante Koeffizienten ................................................... 113

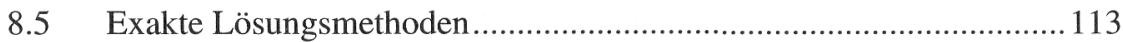

8.5.1 Ansatzmethoden ........................................................ 114

8.5.2 Anwendung der Laplacetransformation ............................. 120

8.5.3 Anwendung von MATHCAD und MATLAB....................... 124

9 Numerische Lösung gewöhnlicher Differentialgleichungen.................... 127

9.1 Einführung......................................................................... 127

9.2 Lösung von Anfangs- und Randwertaufgaben ................................. 131

9.3 Anwendung von MATHCAD und MATLAB .............................. 131

9.4 Anwendung weiterer Programmsysteme ........................................ 132

10 Numerische Lösung von Anfangswertaufgaben gewöhnlicher

Differentialgleichungen ......................................................................................... 133

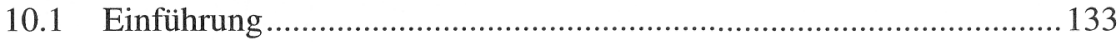

10.2 Diskretisierungsmethoden ...................................................... 134

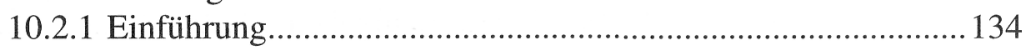

10.2.2 Einschrittmethoden................................................. 135

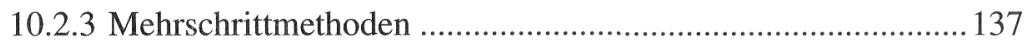

10.2.4 Extrapolationsmethoden ............................................... 138

10.3 Methoden für steife Differentialgleichungen................................. 138

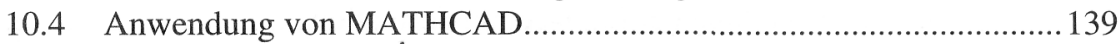

10.5 Anwendung von MATLAB ....................................................... 152

11 Numerische Lösung von Randwertaufgaben gewöhnlicher

Differentialgleichungen ......................................................................... 157

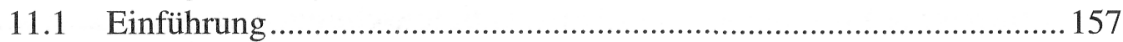

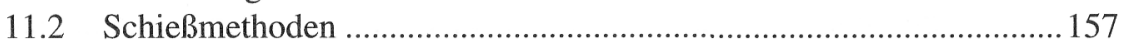

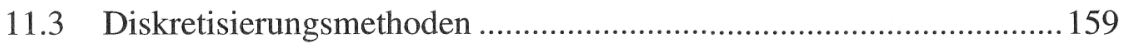

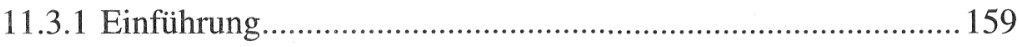

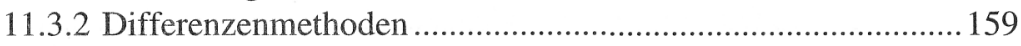

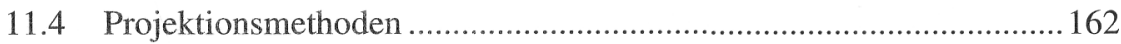

11.4.1 Einführung............................................................. 162

11.4.2 Kollokationsmethoden ................................................... 164

11.4.3 Variationsmethoden: Galerkin-, Ritz- und Finite-ElementeMethoden................................................................ 164

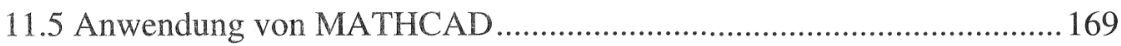

11.6 Anwendung von MATLAB ...................................................... 174

12 Integralgleichungen ...................................................................................... 179

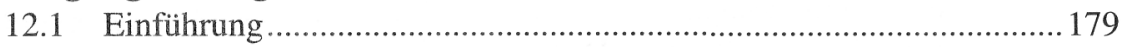

12.2 Integralgleichungsmethode .............................................. 181

13 Partielle Differentialgleichungen .............................................................183

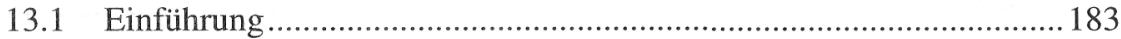

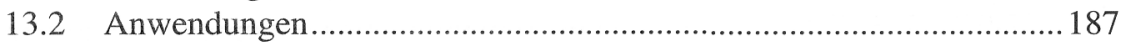

13.3 Anfangs-, Rand- und Eigenwertaufgaben.................................... 190

13.4 Existenz und Eindeutigkeit von Lösungen ................................... 192 


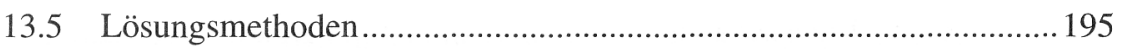

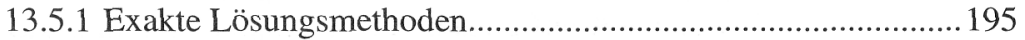

13.5.2 Numerische Lösungsmethoden ..............................................195

13.5.3 Anwendung von MATHCAD und MATLAB ...........................196

13.5.4 Anwendung weiterer Programmsysteme.................................196

14 Partielle Differentialgleichungen erster Ordnung ....................................... 197

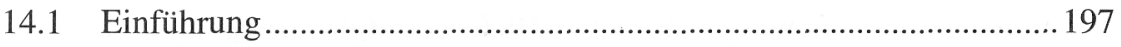

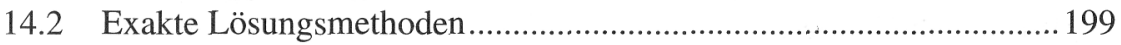

14.2.1 Linear-homogene Differentialgleichungen.............................199

14.2.2 Quasilineare Differentialgleichungen...................................203

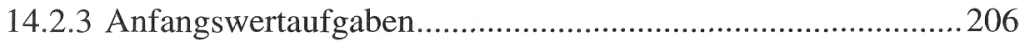

14.2.4 Anwendung von MATHCAD und MATLAB.........................208

15 Partielle Differentialgleichungen zweiter Ordnung.....................................209

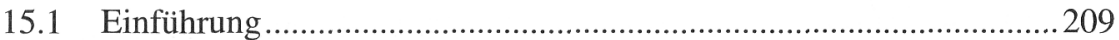

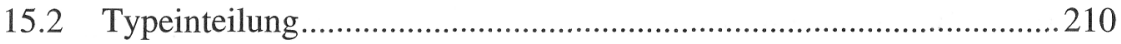

15.3. Anfangs-, Rand- und Eigenwertaufgaben..........................................213

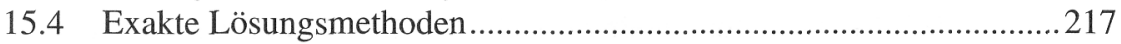

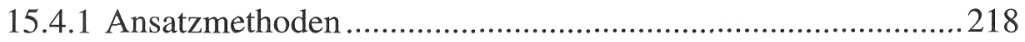

15.4.2 Methode von d'Alembert .....................................................221

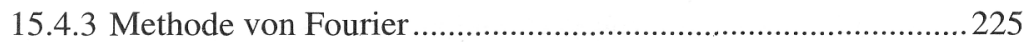

15.4.4 Anwendung der Laplacetransformation .................................2232

15.4.5 Anwendung der Fouriertransformation ..................................2236

15.4.6 Weitere Methoden .................................................................239

15.4.7 Anwendung von MATHCAD und MATLAB.........................241

16 Numerische Lösung partieller Differentialgleichungen.................................243

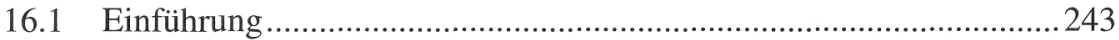

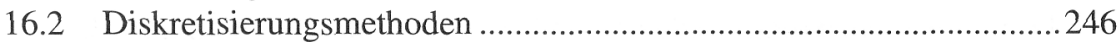

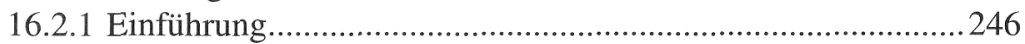

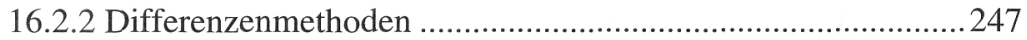

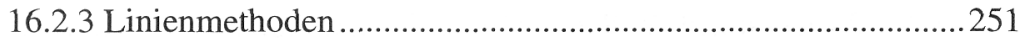

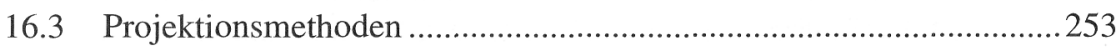

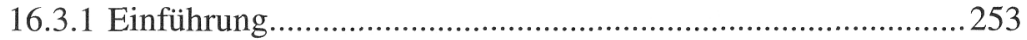

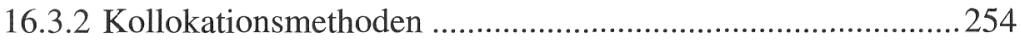

16.3.3 Variationsmethoden: Galerkin-, Ritz- und Finite-Elemente

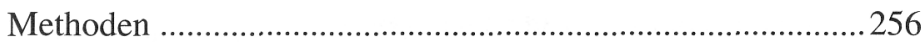

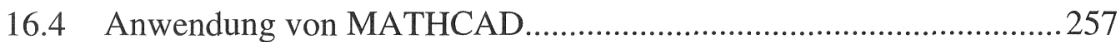

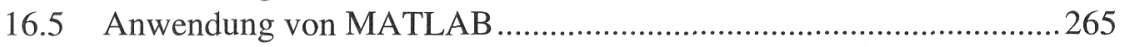

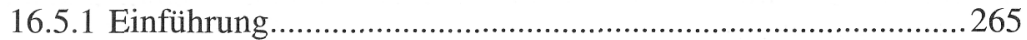

16.5.2 Toolbox Partielle Differentialgleichungen .............................269

16.6 Anwendung weiterer Programmsysteme ............................................2. 276

17 Zusammenfassung ...............................................................................................277

Literaturverzeichnis ................................................................................................281

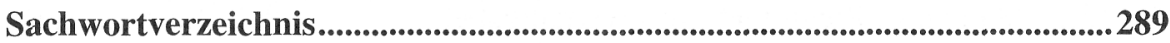

